\title{
Comparison of limberg flap and oval flap techniques in sacrococcygeal pilonidal sinus disease surgery
}

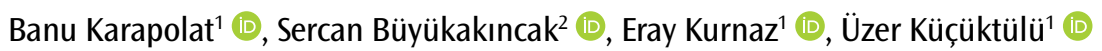

ABSTRACT

ORCID IDs of the authors:

B.K. 0000-0001-5132-8266;

S.B. 0000-0002-1262-9936;

E.K. 0000-0002-9043-558X.

Ü.K. 0000-0003-4481-9176.

\section{Cite this paper as:} Karapolat B, Büyükakıncak S, Kurnaz E, Küçüktülü Ü. Comparison of limberg flap and oval flap techniques in sacrococcygeal pilonidal sinus disease surgery. Turk J Surg 2018; 34(4):311-314.

'Department of General Surgery, Kanuni Training and Research Hospital, Trabzon, Turkey

2Department of General Surgery, Akcaabat State Hospital, Trabzon Turkey

\section{Corresponding Author}

Banu Karapolat

e-mail:banukarapolat@hotmail.com

Received: 23.08 .2017

Accepted: 13.10.2017

Available Online Date: 28.08.2018

CCopyright 2018

by Turkish Surgical Association

Available online at

turkjsurg.com
Objective: Although many surgical techniques have been described for treatment of pilonidal sinus disease (PSD), the ideal treatment method remains controversial. The purpose of this study was to compare Limberg flap and oval flap techniques in patients with PSD.

Material and Methods: Patients diagnosed with PSD who underwent surgery using either the Limberg flap or oval flap technique between January 2012 and January 2016 at the general surgery outpatient clinic were retrospectively reviewed from the database of our hospital; 142 patients (124 males and 18 females) were invited for examination. The demographic characteristics of the patients such as age and gender, hospital stays, seroma occurrence, surgical site infections, wound dehiscence, flap necrosis, loss of sensation, and recurrences were evaluated based on the information obtained from the database and from physical examinations as well as questioning of the patients. The results were statistically compared, and a $p$ value of $<0.05$ was considered significant.

Results: The mean age of the patients was $27.5 \pm 7.8$ years in the Limberg flap group and $26.5 \pm 7.2$ years in the oval flap group. No significant difference was found between the two groups regarding patients' mean age; gender distribution; postoperative hospital stay; recurrence; and complications, such as seroma, infection, wound dehiscence, and loss of sensation. Flap necrosis was not observed in any of the patients.

Conclusion: The Limberg flap and oval flap procedures both involve minimum morbidity and short hospital stay because they were not superior to one another regarding treatment effectiveness, complications, and recurrence in the pilonidal sinus surgery.

Keywords: Pilonidal sinus, surgical flaps, postoperative complications, recurrence

\section{INTRODUCTION}

Many conservative and surgical methods have been described for the treatment of sacrococcygeal pilonidal sinus disease (PSD), but the standard and optimal surgical method remains controversial (1-3). The main principle in the treatment of PSD is to enable patients to return to their normal life at the earliest after a complication-free surgical procedure as well as to prevent recurrence. The medical treatments include local curettage, phenol injection, silver nitrate applications, and electrocauterization of the cavity. The most frequently used surgical methods include excision-primary repair, excision-marsupialization, excision-leaving open, Bascom's operation, Karydakis operation, VYZ plasty, and Limberg flap and oval flap techniques. In recent years, various flap techniques have become popular worldwide, particularly because of low complication and recurrence rates and early wound healing associated with them (2).

In the present study, we compared the Limberg flap and oval flap techniques used in the surgical treatment of PSD with respect to the demographic characteristics of the patients as well as associated complications and recurrence; furthermore, we have discussed the results by referring to the available data.

\section{MATERIAL AND METHODS}

In this study, we included 213 patients who had visited the general surgery outpatient clinic of the Trabzon Kanuni Training and Research Hospital between January 2012 and January 2016 and who had undergone surgery using either the Limberg flap or oval flap technique after being diagnosed with PSD. All the patients were retrospectively reviewed from the database of our hospital, and 142 patients (124 males and 18 females) who could be contacted were invited for examination. The study included patients aged $>18$ years who were diagnosed with chronic PSD, were symptomatic, had not benefited from medical treatment, and did not have any comorbid medical conditions. The exclusion criteria included age $<18$ years, presence of concurrent abscess formation or active infections, recurrent PSD, more complex PSD (orifices of the sinus extending laterally or near the anus), collagen tissue disease, and insufficient medical records. The time elapsed from the surgery to the date of the invitation ranged between 1.5 years and 5 years. The patients were divided into two groups according to the surgical method: Limberg flap and oval flap groups. There were 60 male and 10 female patients in the Limberg flap group and 64 male and 8 female patients in the oval flap group. The mean follow-up period was 31.4 months (range, 18-54 months) in the Limberg flap group and 34.6 months (range 22-60 months) in the oval flap group. The demographic characteristics of the patients such as age and gender, hospital stay, seroma occurrence, surgical site in- 
fections, wound dehiscence, flap necrosis, loss of sensation, and recurrences were evaluated based on the information obtained from the database as well as from physical examinations and questioning of the patients.

The protocol of this study was approved by the local ethics committee, and all the participants provided written informed consent for participation after adequate verbal information was provided to them about the study. The study was conducted in accordance with the principles of the Helsinki Declaration revised in 2000 .

\section{Surgical technique}

All the patients were admitted to the hospital the night before their surgery and treated with two enemas: one at night and the other $2 \mathrm{~h}$ preoperatively. The gluteal and sacral regions of the patients were shaved on the surgery day. All the patients were prophylactically administered $1 \mathrm{~g}$ of ampicillin-sulbactam during anesthesia induction. Under spinal anesthesia, the patients were placed in the prone jackknife position and their gluteus muscles were separated from each other, with a plaster revealing the intergluteal sulcus. To reveal the pilonidal sinus cavity, each patient was injected $2 \mathrm{~mL}$ of methylene blue through the sinus using a 5 $\mathrm{mL}$ hypodermic needle. Marking of healthy tissues was avoided
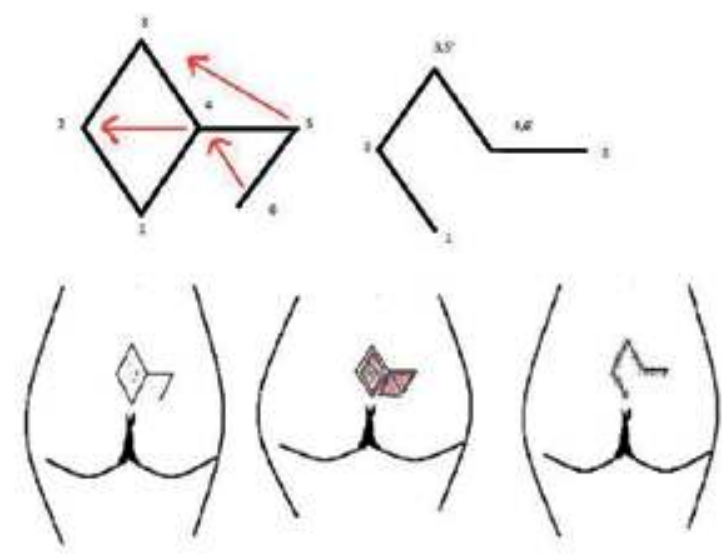

Figure 1. Illustration of Limberg flap preparation
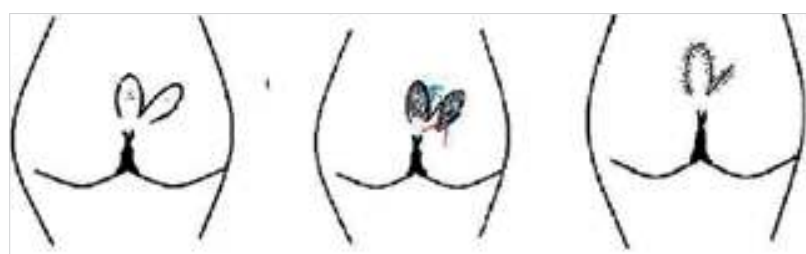

Figure 2. Illustration of oval flap preparation

Table 1. Postoperative complications and recurrence rates

\begin{tabular}{|lccc|}
\hline & Limberg flap & Oval flap & $\mathbf{p}$ \\
\hline Seroma & $5(7.1)$ & $2(2.8)$ & 0.230 \\
\hline Infection & $5(7.1)$ & $2(2.8)$ & 0.230 \\
\hline Wound dehiscence & $7(10)$ & $2(2.8)$ & 0.077 \\
\hline Flap necrosis & $0(0)$ & $0(0)$ & - \\
\hline Loss of sensation & $5(7.1)$ & $5(6.9)$ & 0.594 \\
\hline Recurrence & $3(4.3)$ & $0(0)$ & 0.129 \\
\hline Datas are presented as n (\%) & & & \\
\hline
\end{tabular}

by ensuring that methylene blue was not injected in a pressurized manner. All surgical interventions were performed by the same team. First, the pilonidal cyst was completely excised up to the presacral fascia $2 \mathrm{~cm}$ lateral to the middle line with an ellipticor rhombus-shaped incision without injuring the pilonidal sinus and without leaving any sinus residue. In the patients who were treated using a Limberg flap, the skin was deepened on the gluteus muscle where the flap was to be raised in the form of a continuation of the rhombus in lengths equal to the sides of the rhombus to encompass the subcutaneous tissue and gluteal muscle fascia for preparing a Limberg flap (Figure 1). In the patients who were treated using an oval flap, an oval-shaped was prepared to encompass the same tissue layers from the right and left gluteal regions (Figure 2). After controlling the bleeding, the plasters that maintained the gluteus muscles in traction were opened to ease the wound closure; the flaps were positioned properly and were sutured using a 2-0 polyglactin suture to encompass the fascia-subcutaneous tissues. In all the patients, a vacuum hemovac drain was placed underneath the flap and onto the fascia with its end taken out from another hole and fixed onto the skin ensuring negative pressure drainage. The skin was closed using 3-0 polypropylene sutures. After cleaning the site with physiological saline solution, the dressing was completed using povidone iodine and sterile gauze bandages. There were no major complications or mortality. Postoperatively, the removed pilonidal sinus was sent for pathological examination without opening.

\section{Postoperative period}

The patients were mobilized on postoperative day 1 , and their physical examinations were performed on postoperative days 1 and 5 . All the patients were given ampicillin-sulbactam 375 mg $2 \times 1$ PO as antibiotics for postoperative 5 days. When the drainage dropped below $20 \mathrm{~mL}$, the drains were withdrawn. The sutures were removed on postoperative day 10 . All the patients were trained at the time of their discharge in maintaining hygiene in the perianal and gluteal regions.

\section{Sample size}

The sample size was determined post hoc. At the end of the study, the early postoperative complication rate was found to be $24.3 \%$ in the Limberg flap group and $8.3 \%$ in the oval flap group. Considering the early postoperative complication prevalence as $24.3 \%$, the sample size was calculated to be 71 for one group at $95 \%$ confidence with $10 \%$ deviation, presuming the type 1 error as 0.05 . The sample size calculation was performed using OpenEpi Version 3 (http://www.openepi. com/SampleSize/SSPropor.htm) software.

\section{Statistical Analysis}

All statistical analyses were performed using the Statistical Package for Social Sciences version 15.0 for Windows package program (SPSS Inc., Chicago, IL, USA). Descriptive statistics were used. For comparisons, independent samples t-test and $X^{2}$ test were used. The significance level was set at $p<0.05$.

\section{RESULTS}

The mean age of the patients was $27.5 \pm 7.8$ years in the Limberg flap group and $26.5 \pm 7.2$ years in the oval flap group. There were 60 males $(85.7 \%)$ and 10 females $(14.3 \%)$ in the Limberg flap group and 64 males (88.9\%) and 8 females $(11.1 \%)$ in the oval flap group. No significant difference was observed between the two groups regarding patients' mean age and gender distribution ( $p=0.438$ and $p=0.570$, respectively). 
Postoperative hospital stay was $2.0 \pm 0.9$ days in the Limberg flap group and $1.9 \pm 0.9$ days in the oval flap group. No significant difference was found between the two groups regarding patients' hospital stays $(p=0.649)$. Regarding complications, seroma developed in five $(7.1 \%)$ patients of the Limberg flap group and two (2.8\%) patients of the oval flap group $(p=0.230)$. Infection was noted in five $(7.1 \%)$ patients of the Limberg flap group and two (2.8\%) patients of the oval flap group ( $p=0.230$ ). There was no significant difference between the two groups regarding seroma accumulation and infection.

Wound dehiscence occurred in seven (10\%) patients in the Limberg flap group and two (2.8\%) patients in the oval flap group ( $p=0.077$ ). Flap necrosis was not seen in any of the patients. Loss of sensation was present in five $(7.1 \%)$ patients in the Limberg flap group and five (6.9\%) patients in the oval flap group ( $p=0.594)$. Recurrence occurred in three (4.3\%) patients in the Limberg flap group; no recurrence was seen in the oval flap group $(p=0.129)$. The median time of recurrences was 8 (range, 3-12) months. No significant difference was found between the groups regarding wound dehiscence, flap necrosis, loss of sensation, and recurrence (Table 1).

However, overall early postoperative complications occurred in the Limberg flap group at a rate of $24.3 \%$ (five seroma, five infection, and seven wound dehiscence cases in 17 patients out of 70) and at a rate of $8.3 \%$ in the oval flap group (two seroma, two infection, and two wound dehiscence cases in 6 patients out of 72). A significant difference was found regarding these between the two groups $(p<0.05)$.

\section{DISCUSSION}

Pilonidal sinus disease is an inflammatory disease mostly observed in young males and manifests as an acute painful or chronic form in the intergluteal region $(1,2)$. The incidence is $0.7 \%$, and it is seen mostly in the 15-30-year age group (2-4). Although PSD has been known for many years and frequently encountered in clinics, there is no consensus on the choice of the ideal surgical technique for its treatment, and various surgical methods are available. Often it becomes necessary to reconstruct the cavity using flaps, especially in complicated pilonidal sinuses requiring wide excision. In this study, we compared the Limberg flap and oval flap techniques that are commonly preferred among a large variety of flap procedures found in literature.

The facts that majority of the patients who were operated for PSD in this study were males and the mean age was within the second and third decades indicate the natural characteristics of PSD that have been known for years (2-5).

One of the major factors that determine the quality of life in patients operated for PSD is length of hospital stay and the other is time taken for patient to resume routine activities. Generally, these periods are significantly shorter in patients who are treated with flaps than in those who are left for primary closing or secondary healing. The patients who were treated with the Limberg and oval flaps in this study were hospitalized for an average of 2 days and then discharged. This finding is similar to that reported in literature $(1,6,7)$.

Seroma occurrence was higher in patients treated using Limberg flaps; however, the difference in seroma occurrence was not significant between the two groups. The flaps used in PSD surgery are physiologically fed through macro- and microcirculations. The anatomy of macrocirculation is used in the definition and design of flaps. The arteriolar, capillary, venule, and arteriovenous anastomoses at the microcirculation level are the places where cellular metabolism occurs and where perfusion is often controlled (8). This circulation mechanism is partly disturbed in Limberg flaps as the edges of the flap end in sharp angles, leading to a local blood circulation deficit. Because there are no edges in oval flaps, microcirculation is maintained and a homogenous feeding is secured in the whole flap. The information that seroma accumulation will generally be less in flaps that are fed better can explain our finding of less seroma occurrence in patients treated using oval flaps.

Wound infection is a complication that can be seen in PSD patients in the postoperative period and may involve serious consequences. The infection-causing factors include increased bacterial colonization, wound site being close to the anal channel, obesity, hygiene difficulties due to localization of the lesion, and humidity (5). Additionally, the dead space at the surgical site and in the subcutaneous tissue as well as the tension occurring in the skin may be the cause of many complications, including seroma, hematoma, dehiscence, and infection (9). Infections can endanger flap vitality, adversely affect quality of life in patients, and lead to reoperations and sometimes to long-term painful dressings when the patients are left for secondary healing. In the present study, wound infections developed in five patients in the Limberg flap group and in two patients in the oval flap group. Although there was no significant difference between the two groups regarding wound site infection, the occurrence of 2.5 times more infections in the Limberg flap group than in the oval flap group can be explained by the abovementioned microcirculation-related problems associated with Limberg flaps, particularly the contamination occurring due to the flap edges being closer to the anal channel compared with oval flaps.

The postoperative wound site dehiscence that can be seen in PSD patients occurs because the region is a humid and narrow area and the wound sides that are brought together in a strained way during the surgery may split again after the sutures are removed while performing daily activities, such as sitting down and using the toilet. In fact, one of the main reasons for preferring flap techniques in PSD surgery is that they cause far less strain at the wound sides than primary closing. Despite this, wound dehiscence occurred in seven (10\%) patients treated with Limberg flaps in this study. When these patients were questioned, nearly all of them stated that they performed improper exercises that may have caused excessive strain in the region in the early postoperative period and that they overlooked their local hygiene. Wound dehiscence was seen in only two (2.8\%) patients treated with oval flaps. This may be associated with the oval preparation of the distal free end of the flap instead of it being sharp corner. In fact, the suturing of the sharp distal edges may lead to ischemia and necrosis in Limberg flaps due to decreased circulation at the thin corner. The flap vitality can be better, particularly in the distal corners, in the oval flap reconstruction (10). This can explain the occurrence of wound dehiscence more in the patients treated with Limberg flaps in our study.

When the overall early postoperative complications including seroma, infection, and wound dehiscence were assessed, such complications were seen approximately 3 times more in the Limberg flap group than in the oval flap group. This finding, which 
showed a significant difference between the groups, indicates that there were less perioperative complications in the oval flap group. Nevertheless, a large majority of these complications were treated using minimally invasive methods, such as simple aspiration, drainage-antibiotic therapy, and wound dressings.

Flap necrosis that can occur regardless of the flap technique used in a PSD surgery is known to be an important complication requiring reoperation. In essence, flap necrosis is often a preventable complication if the tissue is handled gently when preparing the flap and care is taken to not impair the pedicular structure of the flap. When preparing flaps, we take care not to damage the vascular structures by avoiding excessive dissection, particularly in the pedicular region. We believe that this careful approach is the reason for the absence of flap necrosis in our study.

Postoperative regional loss of sensation was seen in five patients in both the groups but to the extent not causing any distress that would impair the quality of life in the patients. We believe that the loss of sensation occurred at similar rates in the two groups because the pilonidal sinus tissues removed surgically had approximately the same width.

The term "recurrence" is defined as disease symptoms reemerging a while after full recovery of the wound. In addition to a well-applied surgical intervention, small changes that patients should make in their lifestyles ensuring maintenance of intergluteal region hygiene will prevent recurrence. In this study, recurrence was seen in three (4.3\%) patients in the Limberg flap group and in none in the oval flap group. Being the main problem encountered after a PSD surgery, recurrence has been reported at rates between $3 \%$ and $46 \%$ in literature depending on the technique used (2). With the Limberg flap technique, which is increasingly being preferred at many surgical clinics, recurrence rate ranges between $0 \%$ and $5 \%$ and infection rate ranges between $1.2 \%$ and $4.8 \%(4,6)$. Our results are compatible with those reported in literature $(2,4,6)$. The reasons for recurrence generally include the region being humid and in deep localization, the vacuum effect due to hip movements, obesity, and excessive bacterial colonization due to poor hygiene. The criticized points of the Limberg flap technique are undesired cosmetic appearance, necrosis of the flap vertex, and maceration occurring in the skin at the incision site (2). We believe that the Limberg flap technique used by us involves difficulty in maintaining hygiene because the inferior part of the incision and the lower pole of the flap remain in the intergluteal sulcus and the incision area remains humid and is close to the anal channel; this in turn increases the risk of infection, leading to wound site dehiscence and recurrence. Many studies have reported that the defect occurring after the surgery performed using the Limberg flap technique can be closed fully and without straining; thus, wound site infections at an early stage and recurrences at a later stage are seen less, and with a quick healing time, the time required by the patients to return to their routine life is shorter $(4,6)$. We believe that the reason for the absence of recurrence in patients treated with oval flaps is that the inferior part of the incision remains on the lateral side not entering the intergluteal sulcus; thus, it poses no difficulty of hygiene even in obese people.

The limitations of this study include its retrospective design, single-center nature, and a small sample size. After all, this study was a pilot study showing mid-term results in PSD patients who were surgically treated using two different flap techniques. We believe that the results obtained here will be more meaningful when further prospective, randomized, multicenter studies are conducted with larger patient populations.

\section{CONCLUSION}

The Limberg flap and oval flap techniques used in a PSD surgery are not superior to each other regarding treatment effectiveness, complications, and recurrence. Both are highly effective procedures that can be easily applied and enable complete and unstrained closure of the defect that occurs upon the removal of the affected tissue as a whole; both are associated with minimum morbidity and short hospital stay and are tolerated well by the patients.

Ethics Committee Approval: Authors declared that the research was conducted according to the principles of the World Medical Association Declaration of Helsinki "Ethical Principles for Medical Research Involving Human Subjects" (amended in October 2013).

Informed Consent: Written informed consent was obtained from patients who participated in this study.

Peer-review: Externally peer-reviewed.

Author Contributions: Concept - B.K., S.B., U.K.; Design - B.K., E.K., U.K.; Supervision - B.K., S.B., E.K., U.K.; Resource - B.K., S.B., E.K.; Materials - B.K., S.B., E.K., U.K.; Data Collection and/or Processing - B.K., E.K.; Analysis and/ or Interpretation - B.K., S.B., E.K., U.K.; Literature Search - B.K., S.B., E.K.; Writing Manuscript - B.K., S.B., U.K.; Critical Reviews - B.K., S.B., U.K.

Conflict of Interest: The authors have no conflicts of interest to declare.

Financial Disclosure: The authors declared that this study has received no financial support.

\section{REFERENCES}

1. Kucukkartallar T, Tekin A, Vatansev C, Aksoy F, Erenoglu B. The comparison of the results of different operation techniques for pilonidal sinus disease. Genel Tıp Der 2007; 17: 95-97.

2. Sabuncuoglu MZ, Sabuncuoglu A, Dandin O, Benzin MF, Celik G, Sozen I, et al. Eyedrop-shaped, modified Limberg transposition flap in the treatment of pilonidal sinus disease. Asian J Surg 2015; 38: 161-167. [CrossRef]

3. Tardu A, Haslak A, Ozcınar B, Basak F. Comparison of Limberg and Dufourmentel flap in surgical treatment of pilonidal sinus disease. Ulus Cerrahi Derg 2011; 27: 35-40. [CrossRef]

4. Aren A, Sagban AD, Gokce AH. Comparison of Limberg flap and modified Limberg flap in the surgical treatment of Pilonidal sinus. Istanbul Med J 2010; 11: 149-153.

5. Duman K, Gırgın M, Harlak A. Prevalence of sacrococcygeal pilonidal disease in Turkey. Asian J Surg 2017; 40: 434-437. [CrossRef]

6. Mentes O, Bagci M, Bilgin T, Ozgul O, Ozdemir M. Limberg flap procedure for pilonidal sinus disease: results of 353 patients. Langenbecks Arch Surg 2008; 393: 185-189. [CrossRef]

7. Uçar AD, Cartı EB, Oymacı E, Sarı E, Yakan S, Yıldıım M, et al. Recurrent pilonidal disease surgery: Is it second primary or reoperative surgery? Ulus Cerrahi Derg 2015; 32: 162-167.

8. Vedder N.B. Flap physiology. Mathes Plastic Surgery, 2nd edition. Philadelphia: Saunders Elsevier Inc, Vol: 1; 483-506, 2006.

9. Arslan S, Karadeniz E, Ozturk G, Aydinli B, Bayraktutan MC, Atamanalp SS. Modified Primary Closure Method for the Treatment of Pilonidal Sinus. Eurasian J Med 2016 ; 48: 84-89. [CrossRef]

10. Polat C, Gungor B, Karagul S, Buyukakincak S, Topgul K, Erzurumlu K. Is oval flap reconstruction a good modification for treating pilonidal sinuses? Am J Surg 2011; 201: 192-196. [CrossRef] 